\title{
Association between time of discharge from ICU and hospital mortality: a systematic review and meta-analysis
}

\author{
Si Yang ${ }^{\dagger}$, Zheng Wang ${ }^{\dagger}$, Zhida Liu, Jinlai Wang and Lijun Ma*
}

\begin{abstract}
Background: Epidemiological studies have provided inconsistent results on whether intensive care unit (ICU) discharge at night and on weekends is associated with an increased risk of mortality. This systematic review and meta-analysis aimed to determine whether ICU discharge time was associated with hospital mortality.

Methods: The PubMed, Embase, and Scopus databases were searched to identify cohort studies that investigated the effects of discharge from the ICU on weekends and at night on hospital mortality, with adjustments for the disease severity at ICU admission or discharge. The primary meta-analysis focused on the association between nighttime ICU discharge and hospital mortality. The secondary meta-analysis examined the association between weekend ICU discharge and hospital mortality. Odds ratios (ORs) with 95\% confidence intervals (Cls) were pooled using a random-effects model.

Results: We included 14 studies that assessed outcomes for nighttime versus daytime discharges among 953,312 individuals. Of these 14 studies, 5 evaluated outcomes for weekend versus weekday discharges $(n=70,883)$. The adjusted OR for hospital mortality was significantly higher among patients discharged during the nighttime, compared to patients discharged during the daytime (OR 1.31, 95\% Cl 1.25-1.38, $P<0.0001)$, and the studies exhibited low heterogeneity $\left(P^{2}=33.8 \%, P=0.105\right)$. There was no significant difference in the adjusted ORs for hospital mortality between patients discharged during the weekend or on weekdays (OR 1.03, 95\% Cl 0.88-1.21, $P=0.68$ ), although there was significant heterogeneity between the studies in the weekday/weekend analysis $\left(P^{2}=72.5 \%, P=0.006\right)$.

Conclusions: Nighttime ICU discharge is associated with an increased risk of hospital mortality, while weekend ICU discharge is not. Given the methodological limitations and heterogeneity among the included studies, these conclusions should be interpreted with caution, and should be tested in further studies.
\end{abstract}

Keywords: Hospital mortality, Critically ill, ICU, Discharge, Weekend, Nighttime, Systematic review, Meta-analysis

\section{Background}

The in-hospital mortality rate among intensive care patients is $20-30 \%$, and these patients account for $20-$ $50 \%$ of all hospital deaths $[1,2]$. Thus, clinicians, hospital administrators, and policy makers are challenged to reduce hospital mortality among critically ill patients. The greatest risk of death is related to the intensive care unit (ICU) admission, triage, and discharge, with up to $10.8 \%$ of patients dying after being

\footnotetext{
* Correspondence: 13523548112@163.com

${ }^{\dagger}$ Equal contributors

Department of Respiratory and Critical Care Medicine, Zhengzhou University People's Hospital, Zhengzhou, Henan, China
}

discharged from the ICU $[3,4]$. In this context, death after ICU discharge is predicted by a higher acute physiology score, organ or system failure, older age, prolonged hospitalization, discharge destination, and a do-not-resuscitate order [3, 4].

It is only in recent years that the possible relationship between time of discharge from ICU and hospital mortality has been recognized [5]. Nighttime ICU discharge refers to discharge from ICU at night and during out-of-office hours, and is also known as an "out-of-hours discharge", "afterhours discharge", "nighttime transfer", or "night shift transfer". Retrospective and prospective studies from the UK [6], Australia [7-10], Canada [11, 12], and the USA [13] have 
highlighted the risks of adverse outcomes that may be associated with nighttime ICU discharge. These unfavorable outcomes may include greater in-hospital mortality $[6,10]$, a higher unplanned ICU readmission rate $[9,13]$, and prolonged hospitalization [13]. However, several other studies, including the most recent large-scale prospective study, failed to draw similar conclusions $[14,15]$, and similar inconsistencies have been observed in studies of whether weekend discharge is harmful $[8,10,12,14,16]$.

These discrepancies are likely influenced by the local healthcare systems, patient populations, definitions of nighttime or weekend discharge, disease severity at admission or discharge, therapy limitations, sample size, and study design. Nevertheless, no study has comprehensively examined the discrepancies and similarities among these research results.

Evidence-based practical guidelines have cited the existing research data to support their suggestions on ICU discharge time [17, 18]. Unfortunately, the strength of these recommendations is relatively weak. For example, the UK Faculty of Intensive Care Medicine and the Intensive Care Society suggest avoiding nighttime discharge (between 22:00 and 06:59) to reduce mortality and patient discomfort [17], although this suggestion cites only two retrospective studies $[11,12]$. Based on a more comprehensive literature search, the newly revised American Society of Critical Care Medicine ICU practice guidelines recommend avoiding nighttime ICU discharge but not weekend discharge [18]. However, these two practical recommendations were graded as evidence level $2 \mathrm{C}$ (the highest evidence level is $1 \mathrm{~A}$ ), because they were formulated using a consensus review of contradictory research evidence [18].

Thus, there is a need for stronger evidence that combines all relevant data in a quantitative manner. We performed this systematic review and meta-analysis to identify whether nighttime or weekend ICU discharge is associated with hospital mortality.

\section{Methods}

\section{Data sources and search strategy}

Two independent investigators (SY and JW) performed a systematic search, without language or publication type restrictions, of the PubMed, Embase, and Scopus databases from their inception to 1 August 2016. The searches used a combination of the following search terms with the appropriate wildcards and spelling variations: "intensive care unit", "night-shift", "night", "nighttime", "out-ofhours", "evening", "off-hour", "after-hours", "time", "discharge", "transfer", "mortality", and "death".

The search was limited to studies of human adults (Additional file 1: Table S1). Publications in non-English languages (e.g., French, Japanese, or German) were translated by an independent translation service. Additional searches were performed using two clinical trial registries (http://clinicaltrials.gov/ and http:// www.isrctn.com/), and abstracts from major international conferences were manually searched at their official journal websites (Society of Critical Care Medicine: Critical Care Medicine (1998-2015); American Thoracic Society: American Journal of Respiratory and Critical Care Medicine (2009-2016); European Society of Intensive Care Medicine: Intensive Care Medicine (1988-2014); International Symposium on Intensive Care and Emergency Medicine: Critical Care (1997-2016); American College of Chest Physicians: Chest (2003-2015); Australian and New Zealand Intensive Care Society Annual Meeting: Anaesthesia and Intensive Care (1990-2015)).

Articles that were published online ahead of print in major intensive care journals were searched manually. The two investigators also reviewed the reference lists (Additional file 1: Table S1) of the retrieved studies and relevant reviews to identify additional articles $[19,20]$. In instances where further clarification was required, a third investigator (ZW) emailed the corresponding author of the relevant article.

The meta-analysis was pre-specified and performed according to the Preferred Reporting Items for Systematic Reviews and Meta-Analyses (PRISMA) criteria (Additional file 2) [21]. The meta-analysis and systematic review protocol has not been published, and is not registered with the PROSPERO database or the Cochrane Library.

\section{Study selection}

Studies were considered eligible if they fulfilled the following criteria: (1) a cohort study design; (2) a study population of mainly adult patients who were discharged alive from an ICU (general surgical, medical, or mixed) and were grouped into nighttime/daytime discharges and/or weekday/weekend discharges, and the study assessed outcomes for nighttime versus daytime discharges or outcomes for weekend versus weekdays discharges; (3) the primary outcome was hospital mortality among the patients who were discharged from the ICU (according to their nighttime, daytime, weekday, and/or weekend grouping); and (4) the study reported the effect size and 95\% confident interval (CI) with adjustment for disease severity (or data to calculate these results).

Studies were excluded if they fulfilled any of the following criteria: (1) the study population comprised mainly pediatric patients; (2) the study population comprised patients discharged from a high-dependency or step-down unit; (3) there was no control population; (4) the study was not original research; or (5) the study did not provide sufficient information for data extraction and quality assessment (even after contacting the relevant authors). In cases of duplicate publication, we only 
included the most informative and complete study (typically the most recent publication).

Two investigators (SY and JW) independently screened the titles and abstracts of all citations. The full-text articles were retrieved for full-text review if either investigator thought that the citation might fulfill our eligibility criteria. The same two investigators independently evaluated the eligibility of all full-text articles that were selected during the screening process, and the $\kappa$ value (i.e., chance-independent agreement) was found to be 0.82 . Disagreements were resolved through a consensus process in which investigators discussed the reasoning behind their decisions. In all disagreements, one of the investigators realized that they had made an error.

\section{Data extraction and quality assessment}

Data extraction was independently performed by two investigators (SY and ZL); discrepancies were resolved using discussion and consensus. A predefined standardized data extraction form was used to collect data. The following data were collected from each study: the study name, the first author's name, publication year, the study design, the study location, the patients' ages, the patients' sex distribution, the definition of night or weekend, disease severity, adjustments, outcomes, odds ratios (ORs) and 95\% CIs, numbers of patients discharged during the nighttime and the daytime, crude hospital mortality among patients discharged during nighttime and daytime, numbers of patients discharged during the weekend and weekdays, crude hospital mortality among patients discharged during the weekend and weekdays, and total number of patients discharged. We also checked the supplementary files and contacted the study authors in cases where more detailed information was needed.

Because all of the included studies were cohort studies, the Newcastle-Ottawa Scale (NOS) was used to assess study quality (available at: http://www.ohri.ca/programs/ clinical_epidemiology/oxford.asp) [22]. This scale uses a star system to evaluate study quality in three domains: cohort selection (maximum of four stars), comparability (maximum of two stars), and outcome (maximum of three stars). A score of nine stars indicates the highest possible quality. For the present study, we defined high-quality studies as having $>5$ stars (a low risk of bias) and lowquality studies as having $\leq 5$ stars (a high risk of bias) $[23,24]$. Two investigators (SY and ZL) independently performed the quality assessment; discrepancies were resolved using discussion and consensus.

\section{Statistical analysis}

The primary meta-analysis evaluated the association between nighttime ICU discharge and hospital mortality. The secondary meta-analysis evaluated the association between weekend ICU discharge and hospital mortality. The overall estimates were presented as OR and 95\% CI values, which were determined using a random-effects model that accounted for any differences between the studies, even if there was no statistically significant heterogeneity [25].

The individual estimates were used in the sub-analyses for one study [12] that separately reported ORs for weekday night discharge, weekend daytime discharge, weekend night discharge, and weekday daytime discharge. However, to compare nighttime and daytime discharge, we combined the weekend night discharges and weekday night discharges into a single group. An overall estimate for this group was calculated from the available ORs for weekend night discharge and weekday night discharge using a fixed-effects model and the inverse-variance method. To compare weekend and weekday discharge, we combined weekend night discharges and weekend day discharges into another group. An overall estimate for this group was calculated from the available ORs for weekend night discharge and weekend day discharge using a fixed-effects model and the inverse-variance method [26].

Heterogeneity was evaluated using the Cochran $Q$ statistic and $I^{2}$ statistic, which are quantitative measures of inconsistency across studies [27], and heterogeneity was considered statistically significant at $P$ values $<0.1$ or $I^{2}$ values $>50 \%$. Subgroup analyses were performed to examine the potential sources of heterogeneity using prespecified subgroups that included geographical region, study design, and study characteristics (definition of nighttime and the total number of patients discharged).

We also performed post-hoc subgroup analyses according to adjustments for certain confounding factors (adjustment for illness severity at the ICU discharge, adjustment for treatment limitation orders, and adjustment for premature discharge), as these confounding factors might affect the results of our analyses.

Sensitivity analysis was performed to explore the possible causes of any heterogeneity and to estimate the influence of missing studies on the overall estimates by changing the pooling model (from a random-effects model to a fixed-effects model) and using the one-studyout method. Egger linear regression testing was performed to test for publication bias [28]. All statistical analyses were performed using STATA software (version 12.1; StataCorp, College Station, TX, USA), and differences were considered statistically significant at a twotailed $P$ value $<0.05$.

\section{Results}

Study selection and study characteristics

The initial search identified 5259 potentially relevant publications, although 2113 reports were excluded because of duplicate publication. We also excluded 3106 studies based on reviews of the titles and abstracts. Full-text reviews were performed for the remaining 40 studies, and 
we ultimately identified 14 cohort studies for inclusion in the meta-analysis [5-15, 29-31]. The justifications for the study exclusions are shown in Additional file 1: Table S2. The strategies for study identification and study selection are shown in Fig. 1.

The main characteristics of the 14 included studies are shown in Tables 1, 2 and 3. The 14 studies were all published in English between 2000 and 2015. Six studies were performed in Oceania [7-10, 15, 30], four studies were performed in Europe [5, 6, 14, 29], and the other four studies were performed in North America [11-13, 31]. The studies included six single-center studies and eight multicenter studies, and used a retrospective design $(\mathrm{n}=$ 12) or a prospective design $(n=2)$.

Disease severity was reported based on the Acute Physiology and Chronic Health Evaluation (APACHE) II score $(\mathrm{n}=8$ studies), the APACHE III score $(\mathrm{n}=4)$, or the Simplified Acute Physiology Score (SAPS) II $(n=2)$.
In all 14 studies there was adjustment for a wide range of potential confounders, such as age, treatment limitation orders, premature discharge, diagnostic category, and the use of mechanical ventilation. In 11 studies there was adjustment for disease severity on ICU admission [5-12, 14, 30, 31], and in 3 studies adjustment for disease severity on ICU discharge [13, 15, 29]; in 6 studies there was adjustment for treatment limitation orders $[7,13-15,29,30]$, but no such adjustment in the other 8 studies $[5,6,8-12,31]$. In two studies there was adjustment for premature discharge $[5,7]$.

A total of 953,312 patients were included in the metaanalysis, with the study samples ranging from 1654 patients to 710,535 patients. Four studies included $\leq 10,000$ patients $[6,7,10,29]$ and 10 studies included $>10,000$ patients $[5,8,9,11-15,30,31]$. The mean proportion of nighttime discharges in 13 studies was 15.3\% (range 3.6$34.7 \%$ ), and one study did not report this information

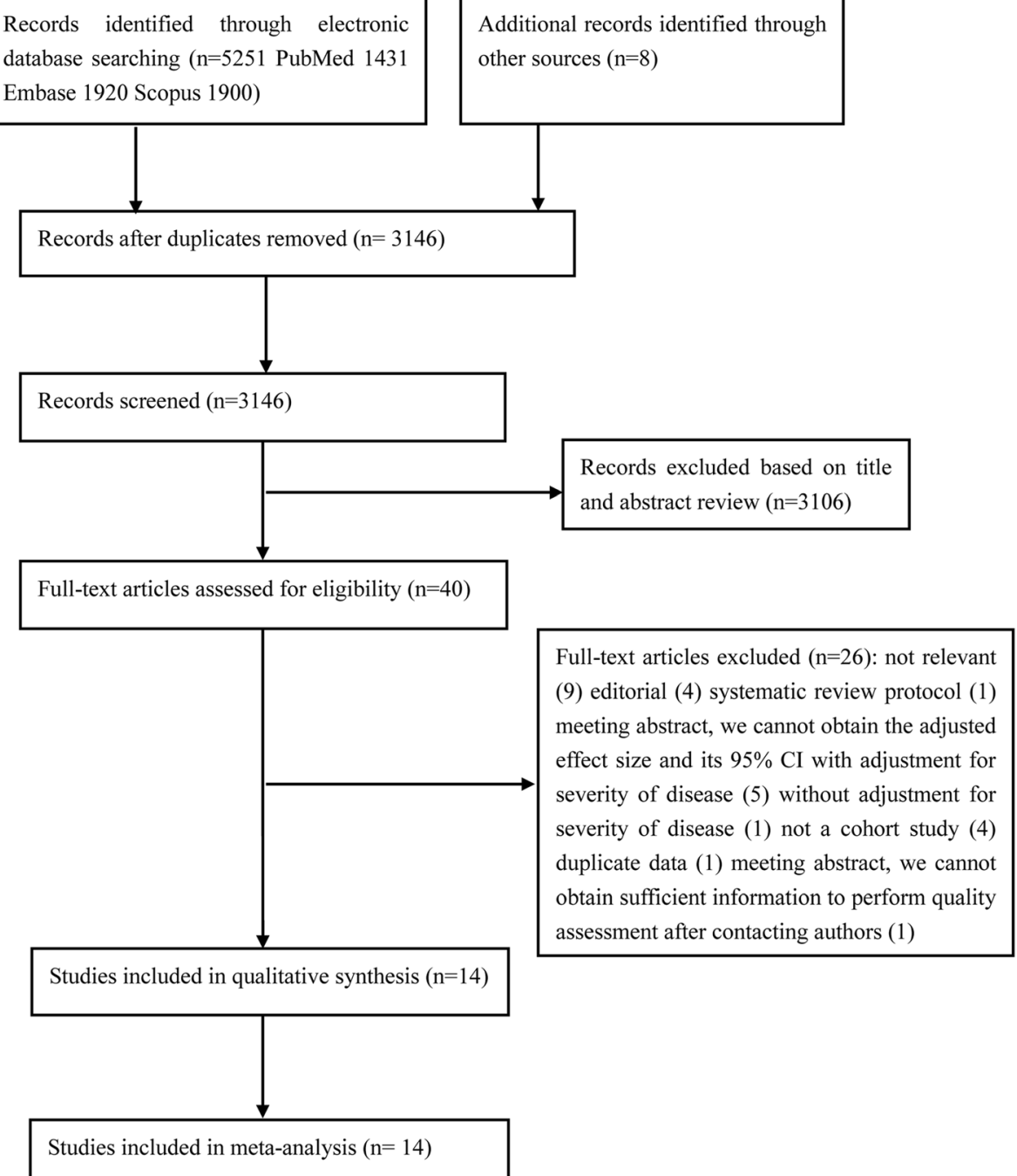

Fig. 1 Flow chart of the article selection process 
Table 1 The main characteristics of the cohort studies included in this meta-analysis

\begin{tabular}{|c|c|c|c|c|c|c|c|c|c|c|c|c|}
\hline $\begin{array}{l}\text { First author/ } \\
\text { publication } \\
\text { year }\end{array}$ & Study location & Study design & Age (years) & Male (\%) & $\begin{array}{l}\text { Definition of } \\
\text { night or } \\
\text { weekend }\end{array}$ & Disease severity & Population & Adjustments & Outcome & OR and $95 \% \mathrm{Cls}$ & $\begin{array}{l}\text { NOS } \\
\text { score }\end{array}$ & Reference \\
\hline $\begin{array}{l}\text { Santamaria } \\
\text { et al./2015 }\end{array}$ & $\begin{array}{l}40 \mathrm{ICUs} \text { in Australia } \\
\text { and New Zealand }\end{array}$ & $\begin{array}{l}\text { Prospective } \\
\text { multicenter } \\
\text { cohort }\end{array}$ & $\begin{array}{l}\text { Median (IQR) } \\
63(49-74)\end{array}$ & 61 & $\begin{array}{l}\text { Night } \\
(18: 00- \\
06: 00)\end{array}$ & $\begin{array}{l}\text { APACHE III-J risk of } \\
\text { death median (IQR) } \\
0.09(0.03-0.25)\end{array}$ & Adult & $\begin{array}{l}\text { Markers of illness severity at } \\
\text { the time of ICU discharge: } \\
\text { age, cardiac surgery, } \\
\text { treatment limitation order, } \\
\text { tracheostomy, ongoing } \\
\text { dialysis, parenteral } \\
\text { nutrition, and altered } \\
\text { conscious state }\end{array}$ & $\begin{array}{l}\text { In-hospital } \\
\text { mortality }\end{array}$ & $1.16(0.89,1.53)$ & 8 & {$[15]$} \\
\hline $\begin{array}{l}\text { Azevedo } \\
\text { et al./2015 }\end{array}$ & 5 ICUs in Canada & $\begin{array}{l}\text { Retrospective } \\
\text { multicenter } \\
\text { cohort }\end{array}$ & $57.5(18.0)$ & 57.9 & $\begin{array}{l}\text { Night (19:00- } \\
\text { 07:59), } \\
\text { weekend (Fri } \\
\text { 19:00-Mon } \\
\text { 07:59) }\end{array}$ & $\begin{array}{l}\text { APACHE II score } \\
19.4(7.5)\end{array}$ & Adult & $\begin{array}{l}\text { Demographics, co-morbidity, } \\
\text { APACHE II score at ICU } \\
\text { admission, use of mechanical } \\
\text { ventilation, ICU length-of- } \\
\text { stay, surgical status, } \\
\text { admission source, primary } \\
\text { diagnostic category, study } \\
\text { year, type of hospital }\end{array}$ & $\begin{array}{l}\text { In-hospital } \\
\text { mortality }\end{array}$ & $\begin{array}{l}\text { Night } 1.29(1.14, \\
1.46) \text {, weekend } \\
0.95(0.84,1.07)\end{array}$ & 6 & [31] \\
\hline $\begin{array}{l}\text { Gantner } \\
\text { et al./2014 }\end{array}$ & $\begin{array}{l}103 \text { ICUs } \\
\text { contributing to } \\
\text { ANZICS APD from } \\
2005 \text { to } 2012\end{array}$ & $\begin{array}{l}\text { Retrospective } \\
\text { multicenter } \\
\text { cohort }\end{array}$ & $\begin{array}{l}\text { After-hours } \\
59.4(19.8), \\
\text { in-hours } 60.3 \\
(19.3)\end{array}$ & NA & $\begin{array}{l}\text { Night (18:00- } \\
06: 00)\end{array}$ & $\begin{array}{l}\text { APACHE III score } \\
\text { After-hours } 50.0 \\
(25.3) \text {, in-hours } \\
46.5(22.9)\end{array}$ & Adult & $\begin{array}{l}\text { APACHE III risk of death at } \\
\text { ICU admission, presence of } \\
\text { treatment limitation orders } \\
\text { at ICU admission, diagnostic } \\
\text { category, hospital site }\end{array}$ & $\begin{array}{l}\text { In-hospital } \\
\text { mortality }\end{array}$ & $1.34(1.30,1.38)$ & 7 & {$[30]$} \\
\hline $\begin{array}{l}\text { Laupland } \\
\text { et al./2011 }\end{array}$ & $\begin{array}{l}\text { French ICUs The } \\
\text { Outcomerea } \\
\text { database }\end{array}$ & $\begin{array}{l}\text { Retrospective } \\
\text { multicenter } \\
\text { cohort }\end{array}$ & $\begin{array}{l}\text { Median (IQR) } \\
62(49-75)\end{array}$ & 61 & $\begin{array}{l}\text { Night 18:00- } \\
07: 59)\end{array}$ & $\begin{array}{l}\text { SAPS II score } \\
\text { median (IQR) } 40 \\
(28-56)\end{array}$ & Adult & $\begin{array}{l}\text { Admission SAPSII, medical/ } \\
\text { surgical classification, } \\
\text { presence of septic shock, } \\
\text { admission decision to forego } \\
\text { life-sustaining therapy(DFLST) } \\
\text { order, discharge SOFA score }\end{array}$ & $\begin{array}{l}\text { In-hospital } \\
\text { mortality }\end{array}$ & $1.54(1.12,2.11)$ & 7 & [29] \\
\hline $\begin{array}{l}\text { Singh } \\
\text { et al./2010 }\end{array}$ & $\begin{array}{l}1 \text { ICU in a tertiary } \\
\text { care teaching } \\
\text { hospital in Australia }\end{array}$ & $\begin{array}{l}\text { Retrospective } \\
\text { single-center } \\
\text { cohort }\end{array}$ & Median 60 & 61.3 & $\begin{array}{l}\text { Night (18:00- } \\
07: 59) \text {, } \\
\text { weekend } \\
\text { (Sat and Sun) }\end{array}$ & $\begin{array}{l}\text { APACHE II score } \\
\text { median } 18 \text { (range, } \\
1-44 \text { ) }\end{array}$ & Adult & $\begin{array}{l}\text { Age, APACHE II score at ICU } \\
\text { admission, discharge } \\
\text { destination }\end{array}$ & $\begin{array}{l}\text { In-hospital } \\
\text { mortality }\end{array}$ & $\begin{array}{l}\text { Night } 1.38(1.01, \\
1.88) \text {, weekend } \\
1.04(0.73,1.46)\end{array}$ & 6 & {$[10]$} \\
\hline $\begin{array}{l}\text { Hanane } \\
\text { et al./2008 }\end{array}$ & $\begin{array}{l}3 \text { ICUs of Mayo } \\
\text { Medical Center } \\
\text { in USA }\end{array}$ & $\begin{array}{l}\text { Retrospective } \\
\text { single-center } \\
\text { cohort }\end{array}$ & $\begin{array}{l}\text { Night } 61.6 \\
(18.0) \text {, day } \\
62.7(17.8)\end{array}$ & $\begin{array}{l}\text { Night } 56.0 \text {, } \\
\text { day } 53.1\end{array}$ & $\begin{array}{l}\text { Night (19:00- } \\
06: 59)\end{array}$ & $\begin{array}{l}\text { APACHE III score } \\
\text { night } 47.6(21.1) \text {, } \\
\text { day } 44.9(19.0)\end{array}$ & Adult & $\begin{array}{l}\text { DNR order by the time of } \\
\text { transfer, the last ICU day } \\
\text { APACHE III predicted } \\
\text { mortality }\end{array}$ & $\begin{array}{l}\text { In-hospital } \\
\text { mortality }\end{array}$ & $1.05(0.64,1.70)$ & 7 & [13] \\
\hline $\begin{array}{l}\text { Laupland } \\
\text { et al./2008 }\end{array}$ & $\begin{array}{l}4 \text { ICUs in the } \\
\text { Calgary Health } \\
\text { Region, Alberta, } \\
\text { Canada }\end{array}$ & $\begin{array}{l}\text { Retrospective } \\
\text { single-center } \\
\text { cohort }\end{array}$ & $\begin{array}{l}\text { Median (IQR) } \\
63.7(49.9- \\
73.8)\end{array}$ & 64 & $\begin{array}{l}\text { Night (18:00- } \\
07: 59) \text {, } \\
\text { weekend (Sat } \\
\text { and Sun) }\end{array}$ & $\begin{array}{l}\text { APACHE II score } \\
25.1(8.48)\end{array}$ & Adult & $\begin{array}{l}\text { Noncardiac surgery, cardiac } \\
\text { surgery, age, APACHE II at } \\
\text { ICU admission, weekend } \\
\text { admission, night admission, } \\
\text { regional resident }\end{array}$ & $\begin{array}{l}\text { In-hospital } \\
\text { mortality }\end{array}$ & $\begin{array}{l}\text { Weekday night } \\
\text { discharge } 1.20 \\
(1.01,1.41), \\
\text { weekend day } \\
\text { discharge } 0.81 \\
(0.67,0.98), \\
\text { weekend night } \\
\text { discharge } 1.35 \\
(1.05,1.73)\end{array}$ & 6 & [12] \\
\hline $\begin{array}{l}\text { Pilcher } \\
\text { et al./2007 }\end{array}$ & $\begin{array}{l}40 \mathrm{ICUs} \text { in Australia } \\
\text { and New Zealand }\end{array}$ & $\begin{array}{l}\text { Retrospective } \\
\text { multicenter } \\
\text { cohort }\end{array}$ & $\begin{array}{l}\text { Night } 58.6 \\
\text { (0.08), day: } \\
59.1(0.17)\end{array}$ & NA & $\begin{array}{l}\text { Night (18:00- } \\
06: 00)\end{array}$ & $\begin{array}{l}\text { APACHE III score } \\
\text { night } 47.7(0.2) \text {, } \\
\text { day: } 46.0(0.1)\end{array}$ & Adult & $\begin{array}{l}\text { APACHE III risk of death at } \\
\text { admission, emergency } \\
\text { admission to ICU }\end{array}$ & $\begin{array}{l}\text { In-hospital } \\
\text { mortality }\end{array}$ & $1.42(1.32,1.53)$ & 6 & [9] \\
\hline
\end{tabular}


Table 1 The main characteristics of the cohort studies included in this meta-analysis (Continued)

\begin{tabular}{|c|c|c|c|c|c|c|c|c|c|c|c|c|}
\hline $\begin{array}{l}\text { Tobin } \\
\text { et al./2006 }\end{array}$ & $1 \mathrm{ICU}$ in Australia & $\begin{array}{l}\text { Retrospective } \\
\text { single-center } \\
\text { cohort }\end{array}$ & 64 (13-98) & 65 & $\begin{array}{l}\text { Night (22:00- } \\
\text { 06:59), } \\
\text { weekend (Fri } \\
\text { 18:00-Mon } \\
\text { 07:59) }\end{array}$ & $\begin{array}{l}\text { APACHE II score } \\
\text { median } 13 \text { (range } \\
0-53 \text { ) }\end{array}$ & Adult & $\begin{array}{l}\text { Age, APACHE II score at } \\
\text { admission, origin of } \\
\text { admission, treatment } \\
\text { category }\end{array}$ & $\begin{array}{l}\text { In-hospital } \\
\text { mortality }\end{array}$ & $\begin{array}{l}\text { Night } 1.63(1.03, \\
2.57) \text {, weekend } \\
1.46(1.18,1.81)\end{array}$ & 6 & {$[8]$} \\
\hline $\begin{array}{l}\text { Priestap } \\
\text { et al./2006 }\end{array}$ & $\begin{array}{l}31 \text { Critical care units } \\
\text { across Canada }\end{array}$ & $\begin{array}{l}\text { Retrospective } \\
\text { multicenter } \\
\text { cohort }\end{array}$ & $\begin{array}{l}\text { Night } 61.6 \\
\text { (17.7), day } \\
61.7(17.5)\end{array}$ & $\begin{array}{l}\text { Night 58, day } \\
57.4\end{array}$ & $\begin{array}{l}\text { Night (21:00- } \\
06: 59)\end{array}$ & $\begin{array}{l}\text { APACHE II score } \\
\text { night } 15.7(7.7) \text {, } \\
\text { day } 15.0(7.4)\end{array}$ & Adult & $\begin{array}{l}\text { Differences in illness severity } \\
\text { at admission, gender, age, } \\
\text { admission source, admission } \\
\text { diagnosis, site }\end{array}$ & $\begin{array}{l}\text { In-hospital } \\
\text { mortality }\end{array}$ & $1.22(1.10,1.36)$ & 6 & [11] \\
\hline $\begin{array}{l}\text { Duke } \\
\text { et al./2004 }\end{array}$ & $\begin{array}{l}1 \mathrm{ICU} \text { in the } \\
\text { Northern Hospital } \\
\text { in Australia }\end{array}$ & $\begin{array}{l}\text { Prospective } \\
\text { single-center } \\
\text { cohort }\end{array}$ & $\begin{array}{l}\text { Median (IQR) } \\
62(42-73)\end{array}$ & NA & $\begin{array}{l}\text { Night (22:00- } \\
07: 30)\end{array}$ & $\begin{array}{l}\text { APACHE \| score } \\
15(10-21)\end{array}$ & Adult & $\begin{array}{l}\text { age, APACHE II predicted } \\
\text { mortality at admission, } \\
\text { premature discharge, } \\
\text { delayed discharge, limitation } \\
\text { of medical treatment decision, } \\
\text { emergency admission, } \\
\text { mechanical ventilation, } \\
\text { APACHE II diagnosis, chronic } \\
\text { health status categories }\end{array}$ & $\begin{array}{l}\text { In-hospital } \\
\text { mortality }\end{array}$ & $1.7(1.03,2.9)$ & 7 & [7] \\
\hline $\begin{array}{l}\text { Uusaro } \\
\text { et al./2003 }\end{array}$ & $\begin{array}{l}18 \text { ICUs in } \\
\text { university and } \\
\text { central hospitals } \\
\text { in Finland }\end{array}$ & $\begin{array}{l}\text { Retrospective } \\
\text { multicenter } \\
\text { cohort }\end{array}$ & NA & NA & $\begin{array}{l}\text { Night (16:00- } \\
\text { 08:00), } \\
\text { weekend (Fri } \\
\text { 16:00-Sun } \\
\text { 24:00) }\end{array}$ & $\begin{array}{l}\text { SAPS \| score } \\
34(17)\end{array}$ & Adult & $\begin{array}{l}\text { Disease severity at ICU } \\
\text { admission, intensity of care, } \\
\text { and whether restrictions for } \\
\text { future care were set }\end{array}$ & $\begin{array}{l}\text { In-hospital } \\
\text { mortality }\end{array}$ & $\begin{array}{l}\text { Night } 1.11(0.93, \\
1.31) \text {, weekend } \\
0.88(0.73,1.07)\end{array}$ & 7 & [14] \\
\hline $\begin{array}{l}\text { Beck } \\
\text { et al./2002 }\end{array}$ & $\begin{array}{l}9 \text { ICUs in a district } \\
\text { general hospital } \\
\text { in United Kingdom }\end{array}$ & $\begin{array}{l}\text { Retrospective } \\
\text { single-center } \\
\text { cohort }\end{array}$ & $57(19)$ & 61.7 & $\begin{array}{l}\text { Night (20:00- } \\
07: 59)\end{array}$ & $\begin{array}{l}\text { APACHE ॥ } \\
\text { probabilities } \\
18.3(18.7)\end{array}$ & Adult & $\begin{array}{l}\text { Disease severity at ICU } \\
\text { admission }\end{array}$ & $\begin{array}{l}\text { In-hospital } \\
\text { mortality }\end{array}$ & $1.70(1.28,2.25)$ & 6 & [6] \\
\hline $\begin{array}{l}\text { Goldfrad } \\
\text { et al./2000 }\end{array}$ & $\begin{array}{l}88 \mathrm{ICUs} \text { in the } \\
\text { United Kingdom }\end{array}$ & $\begin{array}{l}\text { Retrospective } \\
\text { multicenter } \\
\text { cohort }\end{array}$ & $\begin{array}{l}\text { Mean }(95 \% \mathrm{Cl}) \\
\text { night } 57.5 \\
(56.4-58.7) \\
\text { day } 58.2 \\
(57.9-58.5)\end{array}$ & NA & $\begin{array}{l}\text { Night (22:00- } \\
06: 59)\end{array}$ & $\begin{array}{l}\text { APACHE II score } \\
\text { mean }(95 \% \mathrm{Cl}) \text {, } \\
\text { night } 15.5 \\
(15.1-16.0) \text {, day } \\
14.6(14.5-14.7)\end{array}$ & Adult & $\begin{array}{l}\text { Case-mix (age, medical } \\
\text { history, acute severity), } \\
\text { premature discharge }\end{array}$ & $\begin{array}{l}\text { In-hospital } \\
\text { mortality }\end{array}$ & $1.17(0.92,1.49)$ & 6 & [5] \\
\hline
\end{tabular}

Abbreviations: OR odds ratio, Cl confidence interval, ICU intensive care unit, IQR interquartile range, APACHE Acute Physiology and Chronic Health Evaluation, SAPS Simplified Acute Physiology Score, SOFA sequential organ failure assessment, DNR do-not-resuscitate assessment, ANZICS APD Australian and New Zealand Intensive Care Society Adult Patient Database, NOS Newcastle-Ottawa Scale, NA information not available Continuous data given as mean (sd or $95 \% \mathrm{Cl}$ ) or median (interquartile range) if provided by the study authors 
Table 2 Number of patients and crude hospital mortality in studies in which outcomes were assessed for nighttime versus daytime discharge

\begin{tabular}{|c|c|c|c|c|c|}
\hline First author/publication year & $\begin{array}{l}\text { Total number of } \\
\text { patients discharged }\end{array}$ & $\begin{array}{l}\text { Discharged during } \\
\text { daytime, } n(\%)\end{array}$ & $\begin{array}{l}\text { Discharged during } \\
\text { nighttime, } n(\%)\end{array}$ & $\begin{array}{l}\text { Crude hospital } \\
\text { mortality among } \\
\text { patients discharged } \\
\text { during daytime, \% }\end{array}$ & $\begin{array}{l}\text { Crude hospital } \\
\text { mortality among } \\
\text { patients discharged } \\
\text { during nighttime, \% }\end{array}$ \\
\hline Santamaria et al./2015 [15] & 10,211 & $8539(83.6)$ & $1672(16.4)$ & 4.8 & 7.4 \\
\hline Azevedo et al./2015 [31] & 19,622 & $16,117(82.1)$ & $3505(17.9)$ & 8.8 & 11.8 \\
\hline Gantner et al./2014 [30] & 710,535 & $601,151(84.6)$ & $109,384(15.4)$ & 3.6 & 6.4 \\
\hline Laupland et al./2011 [29] & 5992 & $5333(89.0)$ & $659(11.0)$ & 5 & 9 \\
\hline Singh et al./2010 [10] & 1871 & $1221(65.3)$ & $650(34.7)$ & 10.1 & 13.7 \\
\hline Hanane et al./2008 [13] & 11,659 & $11,241(96.4)$ & $418(3.6)$ & 4.5 & 5.3 \\
\hline Laupland et al./2008 [12] & 17,864 & $14,151(79.2)$ & $3713(20.8)$ & 5 & 12 \\
\hline Pilcher et al./2007 [9] & 76,690 & $62,704(81.8)$ & $13,986(18.2)$ & 5.3 & 8 \\
\hline Tobin et al./2006 [8] & 10,903 & NA & NA & NA & NA \\
\hline Priestap et al./2006 [11] & 47,062 & $42,290(89.9)$ & $4772(10.1)$ & 9 & 11.8 \\
\hline Duke et al./2004 [7] & 1870 & $1578(84.0)$ & $292(16.0)$ & 4.3 & 8.2 \\
\hline Uusaro et al./2003 [14] & 20,623 & $16,952(82.2)$ & $3671(17.8)$ & 9.8 & 11.5 \\
\hline Beck et al./2002 [6] & 1654 & $1351(81.7)$ & $303(18.3)$ & 11.2 & 18.8 \\
\hline Goldfrad et al./2000 [5] & 16,756 & $15,747(94.0)$ & $1009(6.0)$ & 13 & 18.1 \\
\hline
\end{tabular}

$N A$ information not available

[8]. The evaluated discharge times included nighttime in all 14 studies [5-15, 29-31] and weekends plus nighttime in 5 studies $[8,10,12,14,31]$. None of the 14 studies used consistent definitions of nighttime or weekend. The average NOS score of the included studies was 6.5 (range 6-8) (see Additional file 1: Table S3).

\section{Nighttime discharge and hospital mortality}

The 14 studies had 953,312 patients who were evaluated for daytime/nighttime discharge (Table 2). The adjusted OR for hospital mortality was significantly higher among patients discharged during the nighttime, compared to patients discharged during the daytime (OR 1.31, 95\% CI 1.25-1.38, $P<0.0001$ ) (Fig. 2), and the individual studies had low heterogeneity $\left(I^{2}=33.8 \%, P=0.105\right)$. We also performed subgroup analyses and sensitivity analyses
(Table 4), which revealed that the significant association between nighttime discharge and hospital mortality was not substantially modified by geographical region, the total number of discharges, or study design (Additional file 3: Figures S1-S3).

Among the 11 studies in which there was adjustment for disease severity on ICU admission, the summary OR for hospital mortality was 1.31 (95\% CI 1.25-1.38). A summary OR was provided for hospital mortality with a broader range (OR 1.26, 95\% CI 1.02-1.57) in the three studies in which there was adjustment for disease severity at ICU discharge (Fig. 3). In our meta-analysis, the risk of hospital mortality did not depend on whether or not the study analyses were adjusted for disease severity at ICU discharge.

Among the six studies in which there was adjustment for treatment limitation orders, the summary OR for

Table 3 Number of patients and crude hospital mortality in studies in which outcomes were assessed for weekend versus weekday discharge

\begin{tabular}{llllll}
\hline First author/publication year & $\begin{array}{l}\text { Total number of } \\
\text { patients discharged }\end{array}$ & $\begin{array}{l}\text { Discharged during } \\
\text { weekend, } n(\%)\end{array}$ & $\begin{array}{l}\text { Discharged during } \\
\text { weekdays, } n(\%)\end{array}$ & $\begin{array}{l}\text { Crude hospital } \\
\text { mortality among } \\
\text { patients discharged } \\
\text { during weekend, \% }\end{array}$ & $\begin{array}{l}\text { Crude hospital } \\
\text { mortality among } \\
\text { patients discharged } \\
\text { during weekdays, } \%\end{array}$ \\
\hline Azevedo et al./2015 [31] & 19,622 & $4676(23.8)$ & $14,946(76.2)$ & NA & NA \\
Singh et al./2010 [10] & 1871 & $567(30.3)$ & $1304(69.7)$ & NA & NA \\
Laupland et al./2008 [12] & 17,864 & $4661(26.1)$ & $13,203(73.9)$ & 6 & 7 \\
Tobin et al./2006 [8] & 10,903 & NA & NA & NA & NA \\
Uusaro et al./2003 [14] & 20,623 & $2932(14.2)$ & $17,691(85.8)$ & 9.2 & 10.2 \\
\hline
\end{tabular}




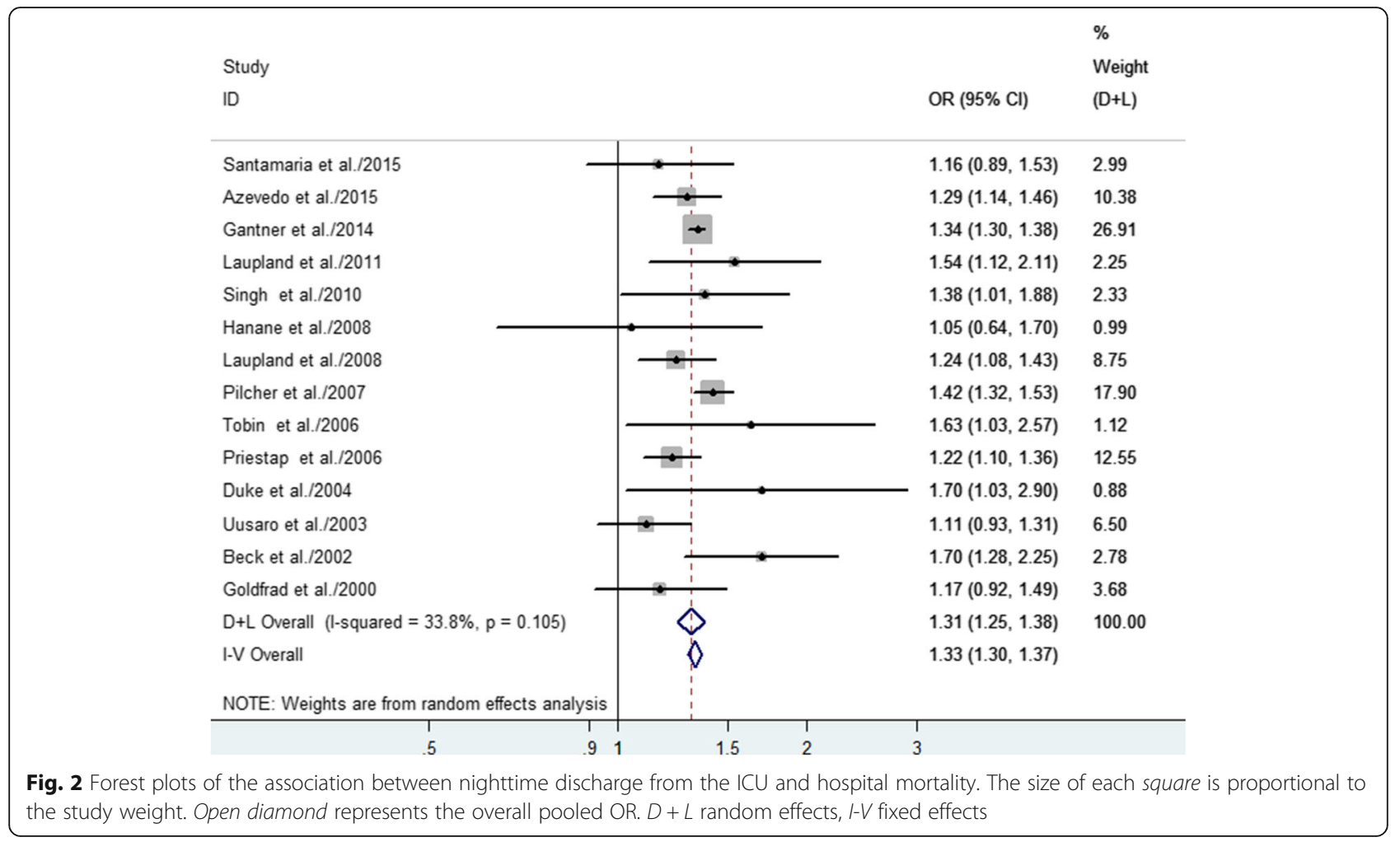

hospital mortality was 1.28 (95\% CI 1.15-1.43); among the remaining eight studies without this adjustment, the summary OR for hospital mortality was 1.32 (95\% CI 1.23-1.42) (Fig. 4). In our meta-analysis, the risk of hospital mortality did not depend on whether or not there was adjustment for treatment limitation orders. We did not observe a significant relationship between nighttime discharge and hospital mortality in the subgroup analysis that was adjusted for premature discharge (Fig. 5). Our sensitivity analyses suggested that the overall estimates were not materially altered by changing the pooling models (random-effects model, OR 1.31, 95\% CI 1.251.38; fixed-effects model, OR 1.33, 95\% CI 1.30-1.37) and were not materially altered when an individual study was omitted from the sequence, with a range of 1.29 (95\% CI 1.22-1.36) to 1.33 (95\% CI 1.27-1.39) (Table 4).

\section{Weekend discharge and hospital mortality}

Five studies with 70,883 patients evaluated weekday/ weekend discharges (Table 3 ). There was no difference in the adjusted ORs for hospital mortality when we compared patients who were discharged during the weekend or on weekdays (OR 1.03, 95\% CI $0.88-1.21, P=0.68$ ) (Fig. 6). However, the studies exhibited significant heterogeneity $\left(I^{2}=72.5 \%, P=0.006\right)$, and we performed sensitivity analyses to explore the possible explanations for the heterogeneity. Our sensitivity analyses suggested that the overall estimates were not materially altered by changing the pooling models (random-effects model, OR 1.03, 95\% CI 0.88-1.21; fixed-effects model, OR 1.00, 95\% CI 0.93-1.08) and were not materially altered when an individual study was omitted from the sequence, with a range of 0.95 (95\% CI $0.87-1.03)$ to 1.08 (95\% CI 0.89-1.31). We were unable to identify the specific study that caused the heterogeneity, which might have been explained by the different patient populations, weekend definitions (including a different number of weekend days and weekend nights), and adjustments for confounding factors. However, when the Tobin study [8] was omitted, the heterogeneity among the remaining four studies was markedly reduced $\left(I^{2}=0.0 \%, P=0.792\right)$, and we did not observe an association between weekend discharge and hospital mortality.

\section{Publication bias}

Egger's test did not reveal any significant evidence of publication bias $(P=0.662$ for nighttime studies, $P=0.507$ for weekend studies) (Additional file 3: Figures S4 and S5).

\section{Discussion}

The present study revealed that nighttime ICU discharge is associated with an increased risk of hospital mortality compared to daytime ICU discharge. Furthermore, it 
Table 4 Subgroup and sensitivity analyses for hospital mortality

\begin{tabular}{|c|c|c|c|c|c|}
\hline Analysis & Studies, $n$ & Odds ratio $(95 \% \mathrm{Cl})$ & $P$ heterogeneity & $R^{2}$ & Study reference \\
\hline \multicolumn{6}{|l|}{ Subgroup analysis } \\
\hline \multicolumn{6}{|l|}{ The definition of night } \\
\hline 18:00-06:00 & 3 & $1.36(1.29,1.43)$ & 0.198 & $38.30 \%$ & {$[9,15,30]$} \\
\hline 18:00-07:59 & 3 & $1.30(1.15,1.46)$ & 0.432 & $0.00 \%$ & {$[10,12,29]$} \\
\hline 19:00-07:59 & 1 & $1.29(1.14,1.46)$ & & & [31] \\
\hline 19:00-06:59 & 1 & $1.05(0.64,1.70)$ & & & [13] \\
\hline 22:00-07:30 & 1 & $1.7(1.03,2.9)$ & & & {$[7]$} \\
\hline 21:00-06:59 & 1 & $1.22(1.10,1.36)$ & & & [11] \\
\hline 16:00-08:00 & 1 & $1.11(0.93,1.31)$ & & & [14] \\
\hline 20:00-07:59 & 1 & $1.70(1.28,2.25)$ & & & {$[6]$} \\
\hline 22:00-06:59 & 2 & $1.30(0.96,1.76)$ & 0.209 & $36.80 \%$ & {$[5,8]$} \\
\hline \multicolumn{6}{|l|}{ Geographic region } \\
\hline Oceania & 6 & $1.35(1.31,1.39)$ & 0.457 & $0.00 \%$ & {$[7-10,15,30]$} \\
\hline Europe & 4 & $1.33(1.08,1.63)$ & 0.039 & $64.00 \%$ & {$[5,6,14,29]$} \\
\hline North America & 4 & $1.24(1.16,1.33)$ & 0.82 & $0.00 \%$ & {$[11-13,31]$} \\
\hline \multicolumn{6}{|l|}{ Total discharge number } \\
\hline$\leq 10000$ & 4 & $1.56(1.32,1.84)$ & 0.785 & $0.00 \%$ & {$[6,7,10,29]$} \\
\hline$>10000$ & 10 & $1.29(1.23,1.36)$ & 0.091 & $39.90 \%$ & {$[5,8,9,11-15,30,31]$} \\
\hline \multicolumn{6}{|l|}{ Study design } \\
\hline Multicenter studies & 8 & $1.30(1.23,1.38)$ & 0.067 & $47.00 \%$ & {$[5,9,11,14,15,29-31]$} \\
\hline Single-center studies & 6 & $1.38(1.20,1.59)$ & 0.268 & $22.10 \%$ & {$[6-8,10,12,13]$} \\
\hline \multicolumn{6}{|c|}{ Whether or not adjusted for severity of illness at the time of ICU discharge } \\
\hline YES & 3 & $1.26(1.02,1.57)$ & 0.296 & $17.80 \%$ & {$[13,15,29]$} \\
\hline NO & 11 & $1.31(1.25,1.38)$ & 0.076 & $40.90 \%$ & {$[5-12,14,30,31]$} \\
\hline \multicolumn{6}{|c|}{ Whether or not adjusted for treatment limitation orders } \\
\hline Yes & 6 & $1.28(1.15,1.43)$ & 0.152 & $38.10 \%$ & {$[7,13-15,29,30]$} \\
\hline No & 8 & $1.32(1.23,1.42)$ & 0.116 & $39.40 \%$ & {$[5,6,8-12,31]$} \\
\hline \multicolumn{6}{|c|}{ whether or not adjusted for premature discharge } \\
\hline Yes & 2 & $1.31(0.94,1.84)$ & 0.2 & $39.20 \%$ & {$[5,7]$} \\
\hline No & 12 & $1.31(1.25,1.38)$ & 0.09 & $37.70 \%$ & {$[6,8-15,29-31]$} \\
\hline \multicolumn{6}{|l|}{ Sensitivity analysis } \\
\hline Fixed-effects model & 14 & $1.33(1.30,1.37)$ & 0.105 & $33.80 \%$ & {$[5-15,29-31]$} \\
\hline Random-effects model & 14 & $1.31(1.25,1.38)$ & 0.105 & $33.80 \%$ & {$[5-15,29-31]$} \\
\hline \multicolumn{6}{|l|}{ One-study-out method } \\
\hline Santamaria et al./2015 & 1 & $1.31(1.24,1.39)$ & & & [15] \\
\hline Azevedo et al./2015 & 1 & $1.31(1.25,1.37)$ & & & [31] \\
\hline Gantner et al./2014 & 1 & $1.31(1.24,1.38)$ & & & [30] \\
\hline Laupland et al./2011 & 1 & $1.30(1.21,1.39)$ & & & [29] \\
\hline Singh et al./2010 & 1 & $1.32(1.25,1.39)$ & & & [10] \\
\hline Hanane et al./2008 & 1 & $1.31(1.25,1.38)$ & & & [13] \\
\hline Laupland et al./2008 & 1 & $1.32(1.25,1.39)$ & & & [12] \\
\hline Pilcher et al./2007 & 1 & $1.31(1.24,1.37)$ & & & [9] \\
\hline Tobin et al./2006 & 1 & $1.29(1.22,1.36)$ & & & {$[8]$} \\
\hline Priestap et al./2006 & 1 & $1.33(1.26,1.40)$ & & & [11] \\
\hline
\end{tabular}


Table 4 Subgroup and sensitivity analyses for hospital mortality (Continued)

\begin{tabular}{llll}
\hline Duke et al./2004 & 1 & $1.32(1.25,1.38)$ & {$[7]$} \\
Uusaro et al./2003 & 1 & $1.31(1.24,1.38)$ & {$[14]$} \\
Beck et al./2002 & 1 & $1.31(1.24,1.38)$ & {$[6]$} \\
Goldfrad et al./2000 & 1 & $1.33(1.27,1.39)$ & {$[5]$} \\
\hline
\end{tabular}

seems that nighttime ICU discharge was also associated with an increased risk of hospital mortality in the subgroup analyses that were stratified according to geographical region, the total number of discharges, or study design (multicenter or single-center).

Patient-related factors, such as disease severity and limitation of medical treatment, are considered crucial determinants of mortality after nighttime ICU discharge $[7,15]$, and disease severity at the ICU admission or discharge is useful for predicting post-ICU mortality. Some of the studies in our meta-analysis found that patients discharged at nighttime were typically older and had more severe injuries, co-morbidities, or multi-organ dysfunction, compared to patients with a daytime discharge, which may explain the higher mortality with nighttime ICU discharge [10, 29, 30]. However, our methodology was initially based on precluding the confounding effect of disease severity. Three studies included adjustment for disease severity at ICU discharge, and the remaining 11 studies included adjustment for disease severity at ICU admission, and both sets had acceptable heterogeneity. After adjusting for disease severity at ICU discharge or admission, there was still a significant relationship between nighttime discharge and hospital mortality (Fig. 3).

Limitation of medical treatment orders refers to limiting or withholding one or more life-support therapies, such as cardiopulmonary resuscitation (do-not-resuscitate orders) or the use of palliative care measures [7]. Patients discharged at nighttime were more likely to have a medical treatment limitation order, which was found to be an independent predictor of mortality in previous studies $[3,7,13,15]$. For example, Santamaria et al. found that treatment limitation orders were an independent predictor of hospital survival (OR 35.4, 95\% CI 27.5-45.6). However, Ouanes et al. observed a

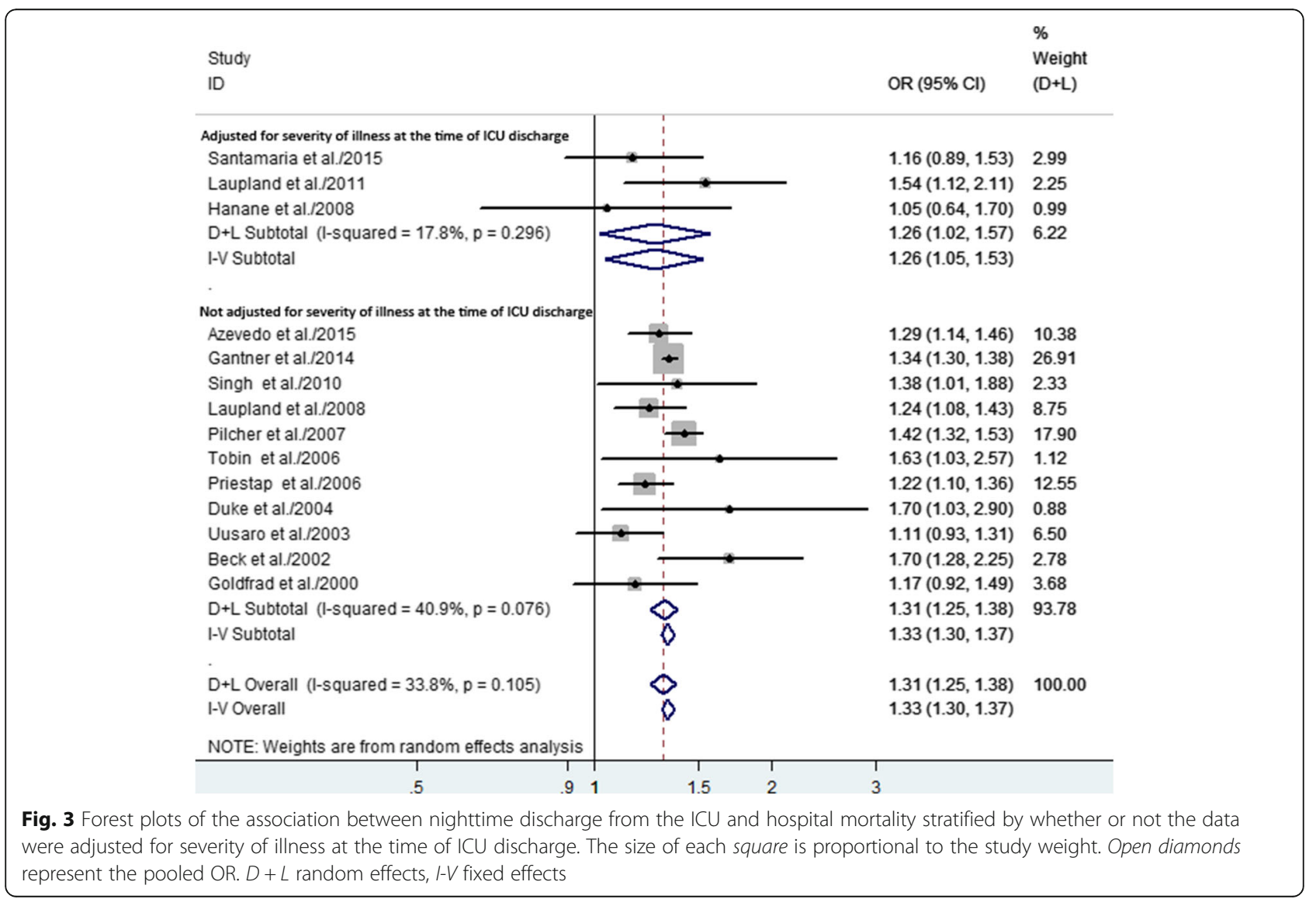




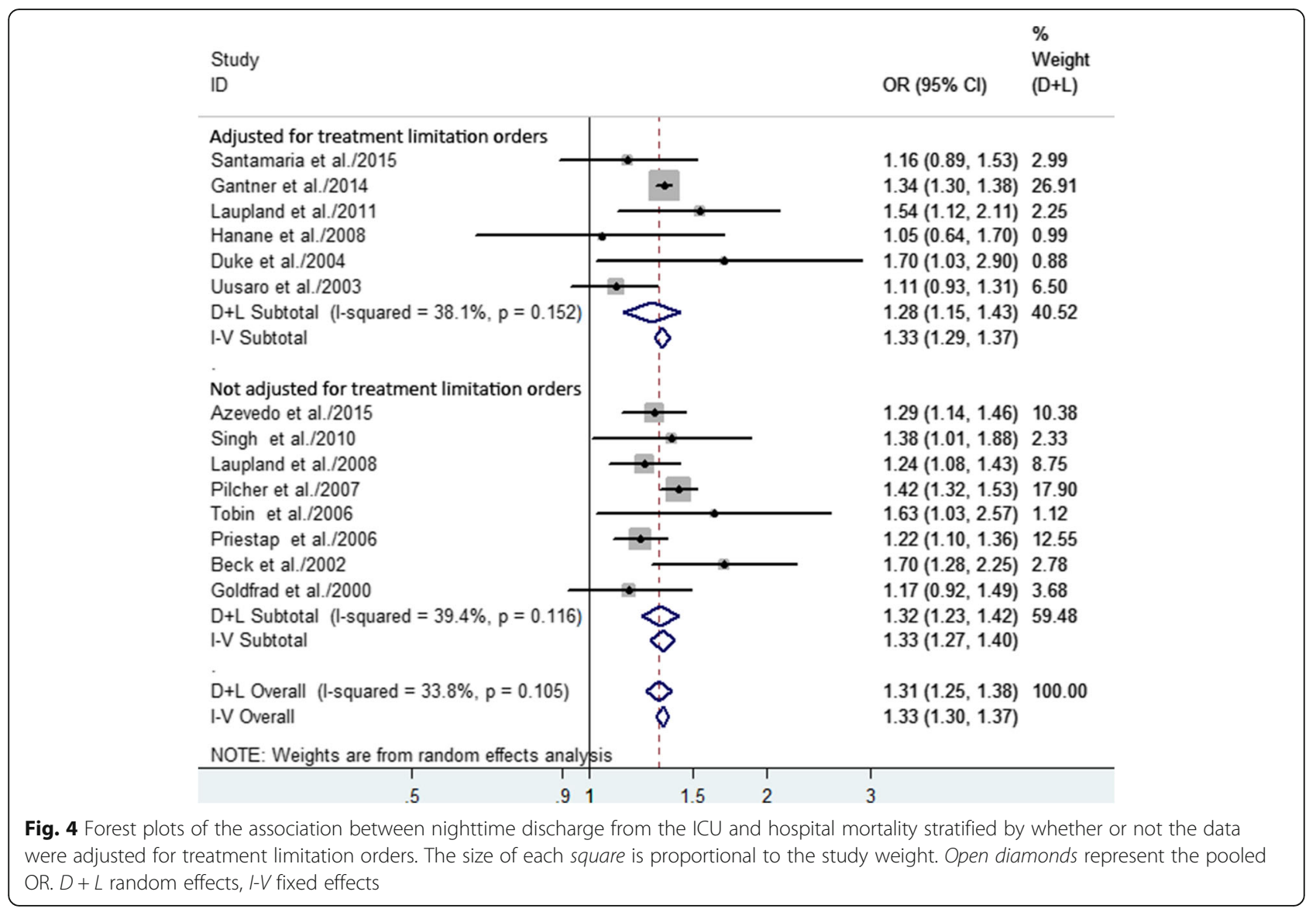

relationship between nighttime discharge and 7-day mortality or readmission after excluding patients with treatment limitation orders [32]. In our meta-analysis, adjustment for treatment limitation orders was documented in six studies, and nighttime discharge remained associated with an increased risk of hospital mortality after the adjustment.

Poor-quality medical care (i.e., lower staffing levels, lower nurse-to-patient ratios, and less surveillance) may partly explain the association between nighttime discharge and increased mortality. Staffing levels and nurse-to-patient ratios in the ICU and general wards are invariably lower at night, and previous studies have reported that increased mortality is associated with decreased staffing levels in the ICU and general wards $[33,34]$. In addition, monitoring devices and life-sustaining devices are considered less immediately available in the general wards [35], and 64\% of major adverse events that occur within $72 \mathrm{~h}$ after ICU discharge may be predicted and prevented by monitoring the patient's vital signs (e.g., an abnormal respiratory rate or tachycardia) [36, 37]. Moreover, transfer to a ward is associated with increased post-ICU mortality, while transfer to a highdependency unit is not, which suggests that patients' outcomes may be associated with the intensity of postICU medical care [6].

There are also systemic factors that may contribute to the post-ICU mortality rate. Practical guidelines recommend that ICU patients should be constantly evaluated for discharge based on their physiological status and the necessity of ICU monitoring [38]. However, in practice, patients are rarely evaluated for discharge at night, unless the ICU is facing pressure from additional admissions. This dilemma may lead to premature discharge, which refers to an unplanned transfer of a patient to make an ICU bed available for a more acutely or seriously sick patient [31].

The proportions of premature discharges vary among different studies based on their defining criteria. Premature ICU discharge was an independent risk factor for mortality in some studies [5, 39], and an additional $48 \mathrm{~h}$ of ICU stay may provide a $39 \%$ reduction in mortality among high-risk patients [40, 41]. Two of the included studies included adjustment for premature discharge, and we did not observe a significant relationship between nighttime discharge and hospital mortality in this subgroup [5, 7]. However, in these two studies (by Goldfrad et al. [5] and Duke et al. [7]) there are conflicting results, and mild 


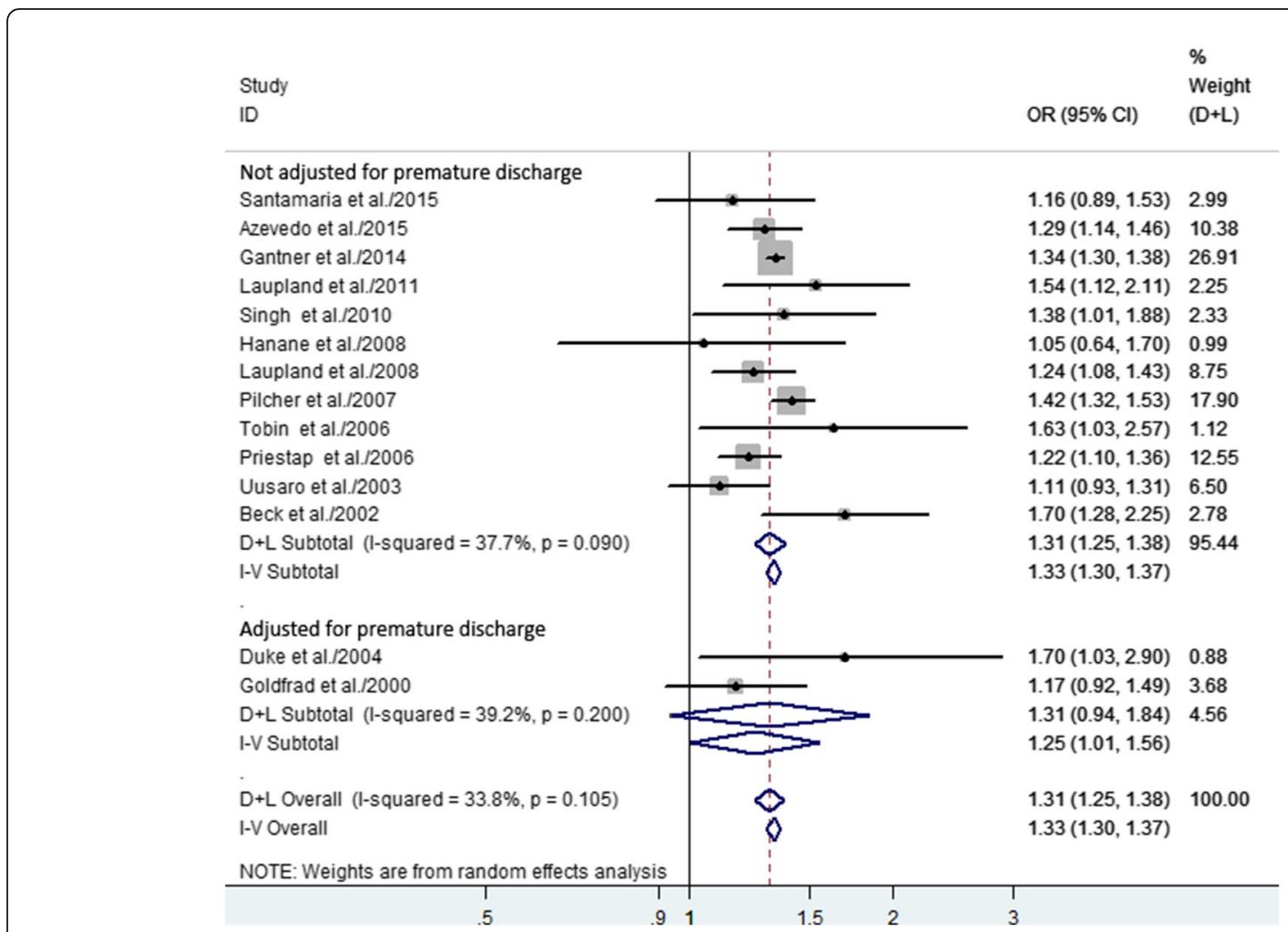

Fig. 5 Forest plots of the association between nighttime discharge from the ICU and hospital mortality stratified by whether or not the data were adjusted for premature discharge. The size of each square is proportional to the study weight. Open diamonds represent the pooled OR. $D+L$ random effects, $I-V$ fixed effects

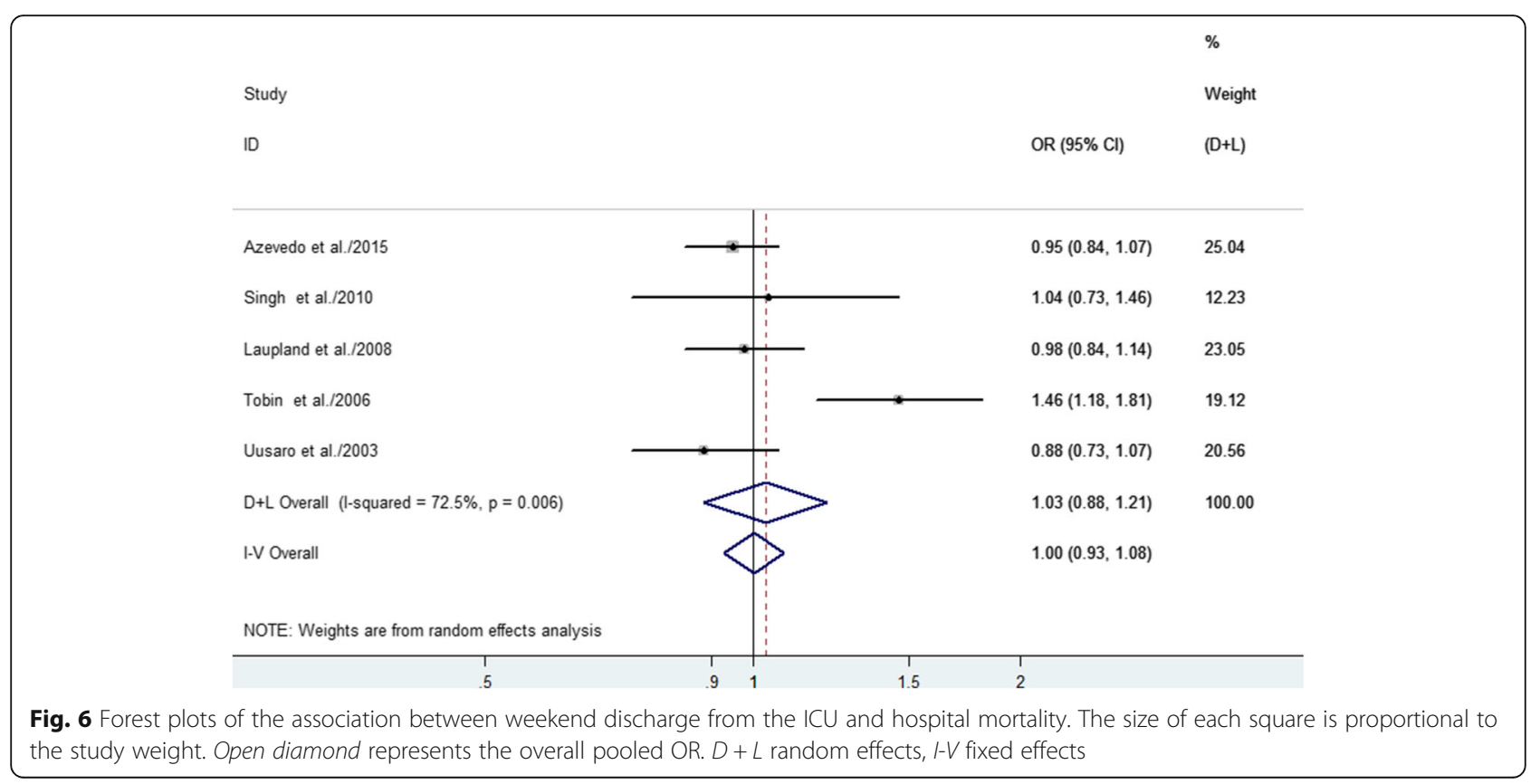


heterogeneity further obscures the results of this subgroup analysis $\left(I^{2}=39.2 \%\right)$. Another study by Santamaria et al. also did not identify an association between premature discharge and subsequent mortality [15]. Thus, the current studies have inconsistent results on whether premature discharge is an independent risk factor for post-ICU mortality, and whether premature discharge affects the association between nighttime ICU discharge and hospital mortality and the magnitude of any related effects remains unclear.

Delayed discharge refers to a planned or prepared discharge that is delayed for some reason, which is commonly a lack of ward beds [42]. Similar to premature discharge, delayed discharge is more likely to occur at night, and each 1-h delay is estimated to be associated with an adjusted 3\% increase in the risk of mortality [43, 44]. Delayed discharge may increase the risk of ICU-acquired infections, which independently influence post-ICU mortality and lead to additional delayed discharges [45].

Both delayed discharge and premature discharge are thought to reflect a limited bed capacity, and some authors even consider nighttime discharge as a marker of a bed shortage $[11,32]$. This may partially reflect the increasing trend in the ratio of nighttime-t-daytime discharge during recent years. Although the average bed occupancy rate in American ICUs has remained fairly constant (1985-2000,65\%; 2000-2010, 68\%), there has been an increase in the demand for intensive care practitioners to provide critical care services [46-48]. Interestingly, improving the number of beds does not always reduce delayed discharge, as Williams et al. found that delayed discharges increased by $4 \%$, despite a significant increase in bed capacity (2000-2001 vs. 2008), which reduced the proportion of "no-bed delays" from $74 \%$ to $36 \%$ [49]. This finding suggests that the problem does not lie in the number of beds, but rather in the inability of the ward to accept patients who are discharged from the ICU in a timely manner. In this scenario, nighttime discharge may be considered an indicator of inefficient patient delivery [50], with nighttime discharge serving to optimize the use of existing ICU beds, instead of simply enlarging the ICU $[15,50]$.

We did not find an association between weekend discharge and hospital mortality, although heterogeneity among the studies may be a question. The different patient populations, weekend definitions (including a different number of weekend days and weekend nights), statistical analyses, and adjustments for confounding factors may also explain the significant heterogeneity. For example, the only positive result (from Tobin et al. [8]) was based on a univariate analysis, while the other studies used a multivariate or logistic regression model. In addition, Tobin et al. defined weekends as being from 18:00 on Friday to 07:59 on Monday (3 days and 3 nights), while the other studies defined weekends as only
Saturday and Sunday [10, 12]. Moreover, the fact that weekend staffing levels and medical care resources are similar to weekdays might explain the negative association between weekend discharge and hospital mortality, although this conclusion remains speculative [12].

\section{Strengths and limitations}

This study has several strengths. First, we identified studies using a comprehensive systematic literature search. Second, we evaluated 953,312 patients with daytime/nighttime discharge, and the large sample size significantly increased the statistical power of the analysis. Third, the pooled estimates were stable after the comprehensive sensitivity analyses. Fourth, we did not detect publication bias, which indicates that the pooled estimates may be unbiased.

This study also has several limitations. First, the cohort studies were observational and descriptive, and we cannot comment on the causality of the relationships between hospital mortality and the factors that we evaluated. Second, the studies used different definitions for nighttime and weekend, which inevitably introduces heterogeneity. Third, although both disease severity at discharge and admission can predict post-ICU mortality, only three of the included studies measured disease severity at ICU discharge. Premature ICU discharge was also more common at night, although only two of the included studies adjusted for premature discharge. Thus, the limited numbers of studies that adjusted for premature discharge or disease severity at ICU discharge might cause residual confounding. Fourth, none of the studies reported the exact demand of care and actual level of care in the ward, which obscures whether or how treatment and nursing care insufficiencies might influence post-ICU mortality. Last, the included studies did not report long-term prognosis or its possible relationship with nighttime discharge, which may also be an important issue. Moreover, the studies failed to address novel trends in critical care, including tele-ICU, liaison nurses, and rapid response teams, which may profoundly alter intensive care and post-ICU care and further alter the rate and effect of nighttime discharge. Therefore, the generalizability of our results in the real world must be tested in future studies [51].

\section{Conclusions}

The present meta-analysis revealed that nighttime ICU discharge was associated with an increased risk of hospital mortality, compared to daytime discharge. Although we did not detect a significant association between hospital mortality and weekend ICU discharge, there was significant heterogeneity among the included studies. Thus, these conclusions should be interpreted with caution, and further large-scale, well-designed, multicenter prospective studies are needed to improve our understanding of the association between ICU discharge times and hospital mortality. 


\section{Additional files}

Additional file 1: Table S1. Database search strategies. Table S2. The justifications for the study exclusions $(n=26)$. Table S3. Newcastle-Ottawa quality assessment of included studies. Table S4. The main characteristics of the excluded meeting abstracts. (DOCX 98 kb)

Additional file 2: PRISMA 2009 checklist. (DOC 62 kb)

Additional file 3: Figure S1. Forest plots of the association between nighttime discharge from the ICU and hospital mortality stratified by geographic region. The size of each square is proportional to the study weight. Open diamonds represent the pooled OR. $D+L$ refers to random effects and $I-V$ to fixed effects. Figure $\mathbf{S}$ 2. Forest plots of the association between nighttime discharge from the ICU and hospital mortality stratified by study design. The size of each square is proportional to the study weight. Open diamonds represent the pooled OR. $D+L$ refers to random effects and $I-V$ to fixed effects. Figure S3. Forest plots of the association between nighttime discharge from the ICU and hospital mortality stratified by the total discharge number. The size of each square is proportional to the study weight. Open diamonds represent the pooled $O R . D+L$ refers to random effects and $I-V$ to fixed effects. Figure $\mathbf{S 4}$. Funnel plots showing the association of nighttime discharge from the ICU with hospital mortality. s.e. refers to standard error, or refers to odds ratio. Figure S5. Funnel plots showing the association of weekend discharge from the ICU with hospital mortality. s.e. refers to standard error, or refers to odds ratio. (ZIP $395 \mathrm{~kb}$ )

\section{Abbreviations}

APACHE: Acute Physiology and Chronic Health Evaluation; Cl: confidence interval; DNR: do-not-resuscitate assessment; ICU: intensive care unit; IQR: interquartile range; NA: information not available; OR: odds ratio; SAPS: Simplified Acute Physiology Score; SOFA: sequential organ failure assessment

\section{Acknowledgements}

Not applicable.

\section{Funding}

This study was supported by the National Natural Science Foundation of China (Grant No.81600047) and the Natural Science Foundation of Henan Province (Grant No.142300410381)

\section{Availability of data and materials}

All data generated or analyzed during this study are included in this published article and its additional files.

\section{Authors' contributions}

SY conceived of and designed the study, participated in the study search, study selection, data collection, and drafted the manuscript. ZW participated in the statistical analysis and interpretation of data and revision of the manuscript. ZDL participated in the data collection and statistical analysis and interpretation of data. JLW participated in the study search, study selection, and statistical analysis and interpretation of data. LJM conceived of and designed the study and revised the manuscript. All authors read and approved the final manuscript.

\section{Competing interests}

The authors declare that they have no competing interests.

\section{Consent for publication}

Not applicable.

\section{Ethics approval and consent to participate}

Not applicable.

Received: 13 May 2016 Accepted: 11 November 2016 Published online: 01 December 2016

\section{References}

1. Knaus WA, Wagner DP, Draper EA, Zimmerman JE, Bergner M, Bastos PG, et al. The APACHE III prognostic system. Risk prediction of hospital mortality for critically ill hospitalized adults. Chest. 1991;100(6):1619-36.

2. Beck DH, Taylor BL, Millar B, Smith GB. Prediction of outcome from intensive care: a prospective cohort study comparing Acute Physiology and Chronic Health Evaluation II and III prognostic systems in a United Kingdom intensive care unit. Crit Care Med. 1997;25(1):9-15.

3. Azoulay E, Adrie C, De Lassence A, Pochard F, Moreau D, Thiery G, et al. Determinants of postintensive care unit mortality: a prospective multicenter study. Crit Care Med. 2003;31(2):428-32.

4. Moreno R, Miranda DR, Matos R, Fevereiro T. Mortality after discharge from intensive care: the impact of organ system failure and nursing workload use at discharge. Intensive Care Med. 2001;27(6):999-1004.

5. Goldfrad C, Rowan K. Consequences of discharges from intensive care at night. Lancet. 2000;355(9210):1138-42.

6. Beck DH, McQuillan P, Smith GB. Waiting for the break of dawn? The effects of discharge time, discharge TISS scores and discharge facility on hospital mortality after intensive care. Intensive Care Med. 2002:28(9):1287-93.

7. Duke GJ, Green JV, Briedis JH. Night-shift discharge from intensive care unit increases the mortality-risk of ICU survivors. Anaesth Intensive Care. 2004;32(5):697-701.

8. Tobin $A E$, Santamaria JD. After-hours discharges from intensive care are associated with increased mortality. Med J Aust. 2006;184(7):334-7.

9. Pilcher DV, Duke GJ, George C, Bailey MJ, Hart G. After-hours discharge from intensive care increases the risk of readmission and death. Anaesth Intensive Care. 2007;35(4):477-85.

10. Singh MY, Nayyar V, Clark PT, Kim C. Does after-hours discharge of ICU patients influence outcome? Crit Care Resusc. 2010;12(3):156-61.

11. Priestap FA, Martin CM. Impact of intensive care unit discharge time on patient outcome. Crit Care Med. 2006;34(12):2946-51.

12. Laupland KB, Shahpori R, Kirkpatrick AW, Stelfox HT. Hospital mortality among adults admitted to and discharged from intensive care on weekends and evenings. J Crit Care. 2008:23(3):317-24.

13. Hanane T, Keegan MT, Seferian EG, Gajic O, Afessa B. The association between nighttime transfer from the intensive care unit and patient outcome. Crit Care Med. 2008;36(8):2232-7.

14. Uusaro A, Kari A, Ruokonen E. The effects of ICU admission and discharge times on mortality in Finland. Intensive Care Med. 2003;29(12):2144-8.

15. Santamaria JD, Duke GJ, Pilcher DV, Cooper DJ, Moran J, Bellomo R. The timing of discharge from the intensive care unit and subsequent mortality. A prospective, multicenter study. Am J Respir Crit Care Med. 2015;191(9):1033-9.

16. Obel N, Schierbeck J, Pedersen L, Storgaard M, Pedersen C, Sørensen HT, et al. Mortality after discharge from the intensive care unit during the early weekend period: A population-based cohort study in Denmark. Acta Anaesthesiol Scand. 2007;51(9):1225-30

17. Faculty of Intensive Care Medicine and the Intensive Care Society: Core standards for intensive care units. 2013;18. https://www.ficm.ac.uk/sites/default/ files/core_standards for icus_ed.1_2013_v2.pdf. Accessed 1 Aug 2016.

18. Nates JL, Nunnally M, Kleinpell R, Blosser S, Goldner J, Birriel B, et al. ICU Admission, discharge, and triage guidelines: a framework to enhance clinical operations, development of institutional policies, and further research. Crit Care Med. 2016:44(8):1553-602.

19. Hosein FS, Roberts DJ, Turin TC, Zygun D, Ghali WA, Stelfox HT. A metaanalysis to derive literature-based benchmarks for readmission and hospital mortality after patient discharge from intensive care. Crit Care. 2014;18(6):715.

20. Elliott M, Bn L, Worrall-Carter, Page K. Factors associated with in-hospital mortality following ICU discharge: a comprehensive review. Carter. 2012;22: $120-5$.

21. Moher D, Liberati A, Tetzlaff J, Altman DG. Preferred reporting items for systematic reviews and meta-analyses: the PRISMA statement. BMJ. 2009;339:b2535.

22. Stang A. Critical evaluation of the Newcastle-Ottawa scale for the assessment of the quality of non randomized studies in meta-analyses. Eur J Epidemiol. 2010;25(9):603-5.

23. Gu WJ, Wang F, Tang L, Liu JC. Single-dose etomidate does not increase mortality in patients with sepsis: a systematic review and meta-analysis of randomized controlled trials and observational studies. Chest. 2015;147(2):335-46.

24. Xiao M, Wang Y, Gao Y. Association between Helicobacter pylori infection and pancreatic cancer development: a meta-analysis. PLoS One. 2013;8(9):e75559. 
25. Furukawa TA, Guyatt GH, Griffith LE. Can we individualize the 'number needed to treat'? An empirical study of summary effect measures in metaanalyses. Int J Epidemiol. 2002;31(1):72-6.

26. Analysing and presenting results. In: Higgins, J. P. T., \& Green, S. (Eds.), Cochrane Handbook for Systematic Reviews of Interventions 4.2.5. 2005. Section 8.

27. Higgins JP, Thompson SG, Deeks JJ, Altman DG. Measuring inconsistency in meta-analyses. BMJ. 2003;327(7414):557-60.

28. Egger M, Davey Smith G, Schneider M, Minder C. Bias in meta-analysis detected by a simple, graphical test. Ibid. 1997;315(7109):629-34.

29. Laupland KB, Misset B, Souweine B, Tabah A, Azoulay E, Goldgran-Toledano D, et al. Mortality associated with timing of admission to and discharge from ICU: a retrospective cohort study. BMC Health Serv Res. 2011;11:321.

30. Gantner D, Farley KJ, Bailey M, Huckson S, Hicks P, Pilcher D. Mortality related to after-hours discharge from intensive care in Australia and New Zealand, 2005-2012. Intensive Care Med. 2014;40(10):1528-35.

31. Azevedo LC, de Souza IA, Zygun DA, Stelfox HT, Bagshaw SM. Association between nighttime discharge from the intensive care unit and hospital mortality: a multi-center retrospective cohort study. BMC Health Serv Res. 2015;15:378

32. Ouanes I, Schwebel C, Français A, Bruel C, Philippart F, Vesin A, et al. A model to predict short-term death or readmission after internsive care unit discharge. J Crit Care. 2012;27:e1-e422. e9.

33. Aiken LH, Clarke SP, Sloane DM, Sochalski J, Silber JH. Hospital nurse staffing and patient mortality, nurse burnout, and job dissatisfaction. JAMA. 2002;288(16):1987-93.

34. West E, Barron DN, Harrison D, Rafferty AM, Rowan K, Sanderson C. Nurse staffing, medical staffing and mortality in intensive care: an observational study. Int J Nurs Stud. 2014;51(5):781-94.

35. Blunt MC, Burchett KR. Out-of-hours consultant cover and case-mix-adjusted mortality in intensive care. Lancet. 2000;356(9231):735-6.

36. McLaughlin N, Leslie GD, Williams TA, Dobb GJ. Examining the occurrence of adverse events within 72 hours of discharge from the intensive care unit. Anaesth Intensive Care. 2007;35(4):486-93.

37. Chaboyer W, Thalib L, Foster M, Ball C, Richards B. Predictors of adverse events in patients after discharge from the intensive care unit. Am J Crit Care. 2008; 17(3):255-63.

38. Task Force of the American College of Critical Care Medicine, Society of Critical Care Medicine. Guidelines for intensive care unit admission, discharge, and triage. Crit Care Med. 1999;27(3):633-8

39. Rodriguez-Carvajal M, Mora D, Doblas A, Garcia M, Dominguez P, Tristancho A, et al. Impact of the premature discharge on hospital mortality after a stay in an intensive care unit. Med Intensiva. 2011;35(3):143-9.

40. Daly K, Beale R, Chang RW. Reduction in mortality after inappropriate early discharge from intensive care unit: logistic regression triage model. BMJ. 2001;322(7297):1274-6

41. Chrusch CA, Olafson KP, McMillan PM, Roberts DE, Gray PR. High occupancy increases the risk of early death or readmission after transfer from intensive care. Crit Care Med. 2009:37(10):2753-8.

42. Williams T, Leslie G. Delayed discharges from an adult intensive care unit. Aust Health Rev. 2004;28(1):87-96.

43. Johnson DW, Schmidt UH, Bittner EA, Christensen B, Levi R, Pino RM. Delay of transfer from the intensive care unit: a prospective observational study of incidence, causes, and financial impact. Crit Care. 2013;17(4):R128. doi:10.1186/ cc12807.

44. Churpek MM, Wendlandt B, Zadravecz FJ, Adhikari R, Winslow C, Edelson DP. Association between intensive care unit transfer delay and hospital mortality: a multicenter investigation. J Hosp Med. 2016;11(11):757-82. doi:10.1002/jhm.2630.

45. Ylipalosaari P, Ala-Kokko TI, Laurila J, Ohtonen P, Syrjala H. Intensive care acquired infection is an independent risk factor for hospital mortality: a prospective cohort study. Crit Care. 2006;10(2):R66.

46. Halpern NA, Pastores SM, Greenstein RJ. Critical care medicine in the United States 1985-2000: an analysis of bed numbers, use, and costs. Crit Care Med. 2004;32(6):1254-9.

47. Halpern NA, Goldman DA, Tan KS, Pastores SM. Trends in critical care beds and use among population groups and medicare and medicaid beneficiaries in the United States: 2000-2010. Crit Care Med. 2016;44(8):1490-9.

48. Kelley MA, Angus D, Chalfin DB, Crandall ED, Ingbar D, Johanson W, et al. The critical care crisis in the United States: a report from the profession. Chest. 2004;125(4):1514-7.
49. Williams TA, Leslie GD, Brearley L, Leen $T$, O'Brien K. Discharge delay, room for improvement? Aust Crit Care. 2010;23(3):141-9.

50. Halpern SD. Nighttime in the intensive care unit. A lens into the value of critical care delivery. Am J Respir Crit Care Med. 2015;191(9):974-5.

51. Tonelli MR, Curtis JR, Guntupalli KK, Rubenfeld GD, Arroliga AC, Brochard L, et al. An official multi-society statement: the role of clinical research results in the practice of critical care medicine. Am J Respir Crit Care Med. 2012;185(10):1117-24.

\section{Submit your next manuscript to BioMed Central and we will help you at every step:}

- We accept pre-submission inquiries

- Our selector tool helps you to find the most relevant journal

- We provide round the clock customer support

- Convenient online submission

- Thorough peer review

- Inclusion in PubMed and all major indexing services

- Maximum visibility for your research

Submit your manuscript at www.biomedcentral.com/submit
Biomed Central 